hep-th/9903038

TAUP-2564-99

\title{
Semi Localized Brane Intersections in SUGRA
}

\author{
A. Loewy \\ School of Physics and Astronomy \\ Beverly and Raymond Sackler Faculty of Exact Sciences \\ Tel Aviv University, Ramat Aviv, 69978, Israel
}

\begin{abstract}
We construct SUGRA solutions of brane configurations of intersecting Dp and NS branes. These solutions are semi-localized in the sense that one of the intersecting branes is smeared along the world volume of the other, while the second is localized. We examine the gauge theory that lives on the localized brane, and the various descriptions possible via $\mathrm{T}$ and $\mathrm{S}$ dualities, and M-theory.
\end{abstract}

\footnotetext{
${ }^{1}$ e-mail: loewy2@post.tau.ac.il
} 


\section{Introduction}

The SUGRA description of intersecting p-branes was considered by many authors [1, 2, 3, 4, 5, 6, 7, 8]. In all these works intersecting branes were smeared in their relative transverse directions. The SUGRA solution was characterized by two harmonic functions of the overall transverse coordinates. Intersection rules have been derived in more than one way to ensure stability, and some unbroken super-symmetry [7, 5]. The metric, dilaton, RR forms

and the NS-NS 2-form are given by a superposition rule [6]. We specialize to the case where a Dp-brane intersects an NS brane over p-1 dimensions.

$$
\begin{aligned}
d s^{2} & =H_{p}^{-1 / 2} d x_{0 . . p-1}^{2}+H_{p}^{1 / 2} d x_{p . .5}^{2}+H_{p}^{-1 / 2} H_{5} d x_{6}^{2}+H_{p}^{1 / 2} H_{5} d x_{789}^{2} \\
e^{2\left(\phi-\phi_{\infty}\right)} & =H_{5} H_{p}^{(3-p) / 2} \\
C_{01 . . p} & =H_{p}^{-1} \quad d B_{2}=* d H_{5}
\end{aligned}
$$

where $H_{p}$ and $H_{5}$ are the Dp and NS brane harmonic functions for $p \leq 4$, $x_{0 . . p-1}$ and $x_{p . .5}$ are the coordinates shared by both branes and the coordinates that belong to the NS brane but not to the Dp-brane respectively. The RR form flux is quantized, and corresponds to the number of Dp-branes. We generalize this construction and allow either $H_{p}$ or $H_{5}$ to depend on relative transverse coordinates as well. The function describing the localized brane should obey a curved space Laplace equation [6, 9, 24], which in the Einstein frame is

$$
g^{\mu \nu} \partial_{\mu} \partial_{\nu} H_{i}=0
$$

Note that the functions will not depend on coordinates in the directions shared by both branes (i.e. 0..p-1). The resulting solution describes a semi localized intersection of two branes. Finding such a solution involves solving the nonlinear differential equation, (2). Solutions of brane intersections in M-theory also obey a similar superposition rule.

SUGRA solutions of various brane constructions are of interest due to the AdS/CFT duality. Intersecting brane configurations were found to be powerful tools in analyzing supersymmetric gauge dynamics in various dimensions [14, 16]. In [18, 19] various brane solutions with 16 supersymmetries and their field theory duals where discussed. By finding SUGRA solutions for intersecting brane configurations one can make statements about field theories with less supersymmetries. The near horizon behavior of such solutions may give us a SUGRA dual of the corresponding field theory. However, if the 
solution is that of a smeared intersection then the field theory data (gauge couplings, energy scale) is not apparent.

In this paper we shall find semi-localized solutions, that is solutions in which one of the harmonic functions depends only on the overall transverse coordinates and the other depends on relative transverse coordinates as well. We shall comment on the field theory duals that these solutions describe. The general form of such solutions was also considered by [11, 12, 24. Some of the solutions that will be presented here have also been obtained by dimensional reduction from 11 to 10 dimensions in the presence of singularities [9, 10, 13].

While finishing this work two related papers by D. Youm hep-th/9902208, and A. Fayyazuddin, D. Smith hep-th/9902210, that contain overlapping results appeared in the electronic archive.

\section{Semi-localized NS branes}

We start with solutions of Dp $\perp$ NS intersections where the NS brane is smeared along the 6 direction, and has its world volume along (12345). We shall think of this configuration as the continuum limit of a periodic array of NS branes along 6, making $H_{5}$ independent of $x_{6}$, see fig.(1). In order that the total number of NS branes be finite, we shall compactify $x_{6}$, and probe this configuration only at distances larger than the compactification radius. We denote by $v$ the coordinates that belong to the NS but not to the Dp-brane, which is extended in $(12 . . \mathrm{p}-1,6)$.

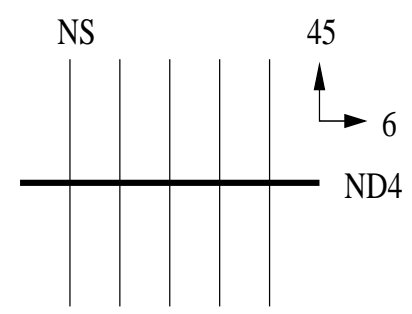

Figure 1: A smeared NS-brane as the continuum limit of a periodic array of NS-branes. The D4-brane is stretched along the compact coordinate 6 . Other Dp-brane configurations are similar. 


\section{$2.1 \quad \mathrm{NS} \perp \mathrm{D} 4$}

We start with an intersection of an NS and D4. The harmonic function describing $N_{5}$ smeared NS branes satisfies $\Delta_{789} H_{5}=0$. This is trivially solved by $1+Q_{5} / r$, where $r^{2}=x_{7}^{2}+x_{8}^{2}+x_{9}^{2}$ (we consider only the spherical symmetric solution for reasons explained below). We shall work in the near horizon limit $H_{5}=Q_{5} / r$. Thus the curved space Laplace equation for the harmonic function describing the $N_{4}$ D4-branes, $H_{4}$ is

$$
\left[\Delta_{45}+H_{5}^{-1} \Delta_{789}\right] H_{4}(v, r)=0
$$

By defining $u=2 \sqrt{Q_{5} r}$, the above equation becomes a sum of two radial Laplace operators in flat space

$$
\left[v^{-1} \partial_{v}\left(v \partial_{v}\right)+u^{-2} \partial_{u}\left(u^{2} \partial_{u}\right)\right] H_{4}(v, u)=0
$$

Eq.(画) can be further simplified by defining $w^{2}=v^{2}+u^{2}$.

$$
\left[w^{-5} \partial_{w}\left(w^{5} \partial_{w}\right)+w^{-2} \Lambda(\lambda)\right] H_{4}(w, \lambda)=0
$$

where $\tan \lambda=u / v$, and $\Lambda(\lambda)$ is the angular part of the Laplace operator. A solution with no $\lambda$ dependence is $H_{4}=1+Q_{4} / w^{4}$ which in the near horizon limit, $Q_{4} \gg w^{4}$, gives according to eq.(1) the following SUGRA solution

$$
\begin{aligned}
d s^{2} & =\frac{w^{2}}{\sqrt{Q_{4}}} d x_{0123}^{2}+\frac{\sqrt{Q_{4}}}{w^{2}} d w^{2}+\sqrt{Q_{4}}\left[d \lambda^{2}+\cos ^{2} \lambda d \beta^{2}+\frac{1}{4} \sin ^{2} \lambda d \Omega_{2}^{2}\right]+\frac{4 Q_{5}^{2}}{\sqrt{Q_{4}} \sin ^{2} \lambda} d x_{6}^{2} \\
e^{2\left(\phi-\phi_{\infty}\right)} & =H_{5} H_{4}^{-1 / 2}=\frac{4 Q_{5}^{2}}{\sqrt{Q_{4}} \sin ^{2} \lambda} \\
R & \sim \frac{1}{\sqrt{Q_{4}}}
\end{aligned}
$$

where $R$ is the curvature of the AdS part of the metric. The field theory described by this configuration should be an $N=2$, four dimensional $\Pi_{i=1}^{N_{5}} S U\left(N_{4}\right)$ supersymmetric gauge theory with $N_{5}$ hyper-multiplets in the bi-fundamental representation, $\left(N_{i}, \bar{N}_{i+1}\right)$, where $i=N_{5}+1$ is identified with $i=1$, since $x_{6}$ is compact. There is also an extra $U(1)$ factor, since this configuration is an elliptic model [15]. This field theory is conformal ( $N_{F}=2 N_{C}$ for each of the gauge groups in the product), as is obvious from the SUGRA solution, which contains an AdS part. We consider solutions 
that depend only on $r, v$ because we wish to preserve the $S O(3)_{r} \times S O(2)_{v}$ isometry, which according to the AdS/CFT duality should be identified with the R-symmetry of the gauge theory.

We now want to write the SUGRA solution in terms of field theory variables, namely the energy scale and $g_{Y M}$.

$$
Q_{4} \sim g_{Y M}^{2} N_{4} \alpha^{\prime 2} \quad Q_{5} \sim N_{5} \alpha^{1 / 2} \quad e^{2 \phi_{\infty}}=g_{s}^{2} \sim g_{Y M}^{4}
$$

Since the field theory is $3+1$ dimensional, $g_{Y M}$ is dimensionless. In order for the metric to have an $\alpha^{\prime}$ factor in front, one should substitute $x_{6}^{2}=\alpha^{\prime} \Phi^{2}$. We also note that there seem to be two energy scales in the solution, $R=r / \alpha^{\prime}$ and $V=v / \alpha^{\prime}$. However, because $v$ and $r$ do not enter symmetrically in the definition of $w$, we cannot at the same time keep $R$ and $V$ fixed when taking $\alpha^{\prime} \rightarrow 0$. We will work only with the combination of the two, $W=w / \alpha^{\prime}$ and $\sin \lambda$. Note, however, that $w=\sqrt{v^{2}+4 Q_{5} r}$ does not have the interpretation of the length of a stretched open string.

The 10 dimensional geometric description is only valid when the effective string coupling $e^{\phi}$, and the curvature of the AdS part in string units are small. Thus as in [19], we can deduce the energies at which we can use this dual description. In this case the dilaton and curvature are only functions of $\sin \lambda$, which means that the geometric description is valid for: $e^{\phi} \ll 1$ and $\alpha^{\prime} R \ll 1$.

$$
e^{2 \phi} \sim \frac{g_{Y M}^{3} N_{5}^{2}}{\sqrt{N_{4}} \sin ^{2} \lambda} \quad \alpha^{\prime} R \sim \frac{1}{g_{Y M} \sqrt{N_{4}}}
$$

When $\sin \lambda \rightarrow 0$ we have two options. One is to elevate this solution to M-theory. We get a configuration of two M5 branes, one of which is smeared over the $(6,10)$ directions, and localized in 789 , and the other localized in all its transverse directions $(45,789)$. This solution is described by the same harmonic functions as before, since the harmonic superposition rule (M5 $\perp$ M5) in 11 dimensions gives the same Laplace equation as the 10 dimensional one.

$$
\begin{aligned}
d s^{2} & =H_{4}^{-1 / 3} H_{5}^{-1 / 3} d x_{0123}^{2}+H_{4}^{2 / 3} H_{5}^{-1 / 3} d x_{45}^{2}+H_{4}^{-1 / 3} H_{5}^{2 / 3} d x_{6,10}^{2}+H_{4}^{2 / 3} H_{5}^{2 / 3} d x_{789}^{2} \\
l_{p}^{2} R & \sim\left[\frac{N_{5}}{N_{4} \sin \lambda}\right]^{2 / 3}
\end{aligned}
$$

This description does not give anything new, since the curvature still diverges when $\sin \lambda \rightarrow 0$. 
We can also go to the T-dual configuration. By making a T duality in the 6 direction we get $N_{4}$ D3 branes smeared in the 6 direction and localized on a singular compact space, $X$. The new metric and dilaton are derived from the original ones using T-duality rules [17].

$$
\begin{aligned}
d s^{2} & =\frac{w^{2}}{\sqrt{Q_{4}}} d x_{0123}^{2}+\frac{\sqrt{Q_{4}}}{w^{2}} d w^{2}+\sqrt{Q_{4}} d s_{X}^{2} \\
e^{2 \phi} & =1
\end{aligned}
$$

where $d s_{X}^{2}$ turns out to be a $Z_{N_{5}}$ orbifold of $S^{5}$, where the orbifold acts on the $S^{3}$ (6789) part of eq.(6). The connection between the NS brane and $A_{N-1}$ singularities, which are described by this metric, and the field content of this theory were discussed in [20, 21, 22, 23]. In the T-dual picture it is possible to generalize the solution to a near extremal brane in the usual way by $g_{00} \rightarrow g_{00} f(w)$ and $g_{w w} \rightarrow g_{w w} f^{-1}(w)$. One can now T-dualize back to the original configuration and obtain the solution for the near extremal brane intersection. This configuration is not expected to be stable. One should note that the radial coordinate $w$ does not have the interpretation of distance from the D3 in transverse space as in Maldacena's original solution [18], since $w^{2}=v^{2}+4 Q_{5} r$.

\section{$2.2 \quad \mathrm{NS} \perp \mathrm{D} 3$}

The next configuration we shall consider is an intersection of $N_{3}$ localized D3 branes with $N_{5}$ NS branes smeared over the 6 direction. The SUGRA solution can be derived in much the same way as in the previous case. We know the near horizon behavior of $H_{5}=Q_{5} / r$ and thus the equation for $H_{3}$ is

$$
\left[\Delta_{345}+H_{5}^{-1} \Delta_{789}\right] H_{3}(v, r)=0
$$

Again we substitute $u=2 \sqrt{Q_{5} r}$, look for solutions that depend only on $u, v$ in order to keep the $S O(3)_{r} \times S O(3)_{v}$ isometry that will correspond to an R-symmetry, and write eq.(11) as a 7 dimensional flat space Laplace operator.

$$
\left[w^{-6} \partial_{w}\left(w^{6} \partial_{w}\right)+w^{-2} \Lambda(\lambda)\right] H_{3}(w, \lambda)=0
$$

The solution that has no $\lambda$ dependence is $H_{3}=1+Q_{3} / w^{5 / 2}$ and we shall work in the near horizon limit. The SUGRA solution follows, as before, from 
eq.(11)

$$
\begin{aligned}
d s^{2} & =\frac{w^{5 / 2}}{\sqrt{Q_{3}}} d x_{012}^{2}+\frac{\sqrt{Q_{3}}}{w^{5 / 2}} d w^{2}+\frac{\sqrt{Q_{3}}}{w^{1 / 2}}\left[d \lambda^{2}+\cos ^{2} \lambda d \Omega_{2}^{2}+\frac{1}{4} \sin ^{2} \lambda d \Omega_{2}^{2}\right]+\frac{4 Q_{5}^{2} w^{1 / 2}}{\sqrt{Q_{3}} \sin ^{2} \lambda} d x_{6}^{2} \\
e^{2\left(\phi-\phi_{\infty}\right)} & =H_{5}=\frac{4 Q_{5}^{2}}{w^{2} \sin ^{2} \lambda} \\
R & \sim \frac{\sqrt{w}}{\sqrt{Q_{3}}}
\end{aligned}
$$

where $R$ is the curvature of the $(012 w)$ part of the metric. Note that, unlike the NS $\perp$ D4 solution, this metric does not describe a product space with an AdS part, thus the corresponding field theory will not be conformal.

The field theory described by this configuration should be a $\Pi_{i=1}^{N_{5}} S U\left(N_{3}\right)$ gauge theory in $\mathrm{d}=2+1$ dimensions with 8 super-symmetries and $N_{5}$ hypermultiplets in the $\left(N_{i}, \bar{N}_{i+1}\right)$ representations. As in the previous case, there is an extra $U(1)$ factor. The field theory description is valid where $g_{\text {eff }}^{2}=$ $g_{Y M}^{2} N_{3} W^{-1}$ is small: $W \gg g_{Y M}^{2} N_{3}$.

The geometric dual is valid when the effective string coupling, as well as, the curvature in string units, are small. To see that we first write the solution in terms of the field theory variables, $W=w / \alpha^{\prime}$ and $N_{3}, N_{5}$.

$$
\begin{gathered}
Q_{3} \sim g_{Y M}^{2} N_{3} \alpha^{\prime 3} \quad Q_{5} \sim N_{5} \alpha^{1 / 2} \quad e^{2 \phi_{\infty}}=g_{s}^{2} \sim g_{Y M}^{4} \alpha^{\prime} \\
\frac{N_{5} g_{Y M}^{2}}{\sin \lambda}<W<g_{Y M}^{2} N_{3}
\end{gathered}
$$

When the string coupling becomes large we can go to the S-dual description. The resulting SUGRA solution is just $H_{5}^{-1 / 2}$ times the original, and the dilaton is just $-\phi$.

$$
\begin{aligned}
d s_{d u a l}^{2} & =H_{5}^{-1 / 2} d s^{2} \\
e^{2 \phi} & =H_{5}^{-1} \sim \frac{W^{2} \sin ^{2} \lambda}{N_{5}^{2} g_{Y M}^{4}} \\
\alpha^{\prime} R & \sim \frac{N_{5} g_{Y M}}{\sqrt{N_{3}} W^{1 / 2}}
\end{aligned}
$$

We can also T-dualize along the 6 direction thus getting $N_{3}$ D2 branes smeared over 6, and localized on a singularity. The resulting SUGRA solution is

$$
d s^{2}=\frac{w^{5 / 2}}{\sqrt{Q_{3}}} d x_{012}^{2}+\frac{\sqrt{Q_{3}}}{w^{5 / 2}} d w^{2}+\frac{\sqrt{Q_{3}}}{w^{1 / 2}} d s_{X}^{2}
$$




$$
e^{2 \phi} \sim \frac{g_{Y M}^{5} \sqrt{N_{3}}}{W^{5 / 2}}
$$

where now $d s_{X}^{2}$ is a $Z_{N_{5}}$ orbifold of $S^{6}$. As in the D4 case, the $Z_{N_{5}}$ orbifold acts only on the $S^{3}$ (6789) part of the transverse space. This solutions resembles the solution found in [19] for D2 branes in flat space, where the original $d \Omega_{6}$ is now orbifolded, and $w$ is not the naive distance from the D2 brane. Again we can generalize to a near extremal solution and T-dualize back to get the near extremal solution of the intersection.

\section{$2.3 \quad \mathrm{NS} \perp \mathrm{D} 2$}

The next configuration we shall discuss is the intersection of $N_{2}$ localized D2 branes with $N_{5}$ NS branes. $H_{5}$ is the same as in the two previous cases, thus the equation for $\mathrm{H}_{2}$ is

$$
\left[w^{-7} \partial_{w}\left(w^{7} \partial_{w}\right)+w^{-2} \Lambda(\lambda)\right] H_{2}(w, \lambda)=0
$$

The solution with no $\lambda$ dependence is $H_{2}=1+Q_{2} / w^{6}$, thus getting the following SUGRA solution

$$
\begin{aligned}
d s^{2} & =\frac{w^{3}}{\sqrt{Q_{2}}} d x_{01}^{2}+\frac{\sqrt{Q_{2}}}{w^{3}} d w^{2}+\frac{\sqrt{Q_{2}}}{w}\left(d \lambda^{2}+\cos ^{2} \lambda d \Omega_{3}^{2}+\frac{1}{4} \sin ^{2} \lambda d \Omega_{2}^{2}\right)+\frac{4 Q_{5}^{2} w}{\sqrt{Q_{2}} \sin ^{2} \lambda} d x_{6}^{2} \\
e^{2\left(\phi-\phi_{\infty}\right)} & =H_{5} H_{2}^{1 / 2}=\frac{4 Q_{5}^{2} \sqrt{Q_{2}}}{w^{5} \sin ^{2} \lambda} \\
R & \sim \frac{w}{\sqrt{Q_{2}}}
\end{aligned}
$$

where now $R$ is the curvature on the $(01 w)$ part of the metric. The field theory is a $1+1$ dimensional $\Pi_{i=1}^{N_{5}} S U\left(N_{2}\right)$ gauge theory, with an extra $U(1)$, and with $N_{5}$ hyper-multiplets, and 8 supersymmetries. It can be described by perturbation theory when $W \gg g_{Y M} \sqrt{N_{2}}$. We can express $Q_{2}, Q_{5}$ in

terms of field theory variables as

$$
Q_{2} \sim g_{Y M}^{2} N_{2} \alpha^{\prime 4} \quad Q_{5} \sim N_{5} \alpha^{\prime 1 / 2} \quad e^{2 \phi_{\infty}}=g_{s}^{2} \sim g_{Y M}^{4} \alpha^{\prime 2}
$$

Thus the geometric description will be valid when

$$
\left[\frac{N_{5}^{2} g_{Y M}^{5} \sqrt{N_{2}}}{\sin ^{2} \lambda}\right]^{1 / 5}<W<g_{Y M} \sqrt{N_{2}}
$$


The solution can be elevated to M-theory, where we find an intersection of a localized M2 and an M5 smeared along $(6,11)$. The solutions is described by the same harmonic functions

$$
\begin{aligned}
d s^{2} & =H_{2}^{-2 / 3} H_{5}^{-1 / 3} d x_{01}^{2}+H_{2}^{1 / 3} H_{5}^{-1 / 3} d x_{2345}^{2}+H_{2}^{-2 / 3} H_{5}^{2 / 3} d x_{6,10}^{2}+H_{2}^{1 / 3} H_{5}^{2 / 3} d x_{789}^{2} \\
l_{p}^{2} R & \sim\left[\frac{N_{5} g_{Y M}}{W \sqrt{N_{2}} \sin \lambda}\right]^{2 / 3}
\end{aligned}
$$

There is also the T-dual description of a D1 on an orbifold singularity given by

$$
\begin{aligned}
d s^{2} & =\frac{w^{3}}{\sqrt{Q_{2}}} d x_{01}^{2}+\frac{\sqrt{Q_{2}}}{w^{3}} d w^{2}+\frac{\sqrt{Q_{2}}}{w} d s_{X}^{2} \\
e^{2 \phi} & =H_{2} \sim \frac{g_{Y M}^{6} N_{2}}{W^{6}}
\end{aligned}
$$

The T-dual solution resembles the solution found for a D1 brane in flat space [19], with the original $d \Omega_{7}$ orbifolded.

\section{$2.4 \quad \mathrm{NS} \perp \mathrm{D} 1$}

The last configuration that we describe in this section has no apparent field theory dual. A D1-brane along the 6 direction intersecting a smeared NS brane is described by the function $H_{1}(v, r)$ that obeys

$$
\left[\Delta_{12345}+H_{5}^{-1} \Delta_{789}\right] H_{1}(v, r)=0
$$

Using similar techniques as in the previous cases, we change variables to $w, \lambda$ and get the following equation

$$
\left[w^{-8} \partial_{w}\left(w^{8} \partial_{w}\right)+w^{-2} \Lambda(\lambda)\right] H_{1}(w, \lambda)=0
$$

A solution with no $\lambda$ dependence gives the following metric

$$
\begin{aligned}
d s^{2} & =\frac{w^{7 / 2}}{\sqrt{Q_{1}}} d x_{01}^{2}+\frac{\sqrt{Q_{1}}}{w^{7 / 2}} d w^{2}+\sqrt{Q_{1}}\left(d \lambda^{2}+\cos ^{2} \lambda d \Omega_{4}^{2}+\frac{1}{4} \sin ^{2} \lambda d \Omega_{2}^{2}\right)+\frac{4 Q_{5}^{2} w^{3 / 2}}{\sqrt{Q_{1}} \sin ^{2} \lambda} d x_{6}^{2} \\
e^{2\left(\phi-\phi_{\infty}\right)} & =H_{5} H_{1}=\frac{4 Q_{5}^{2} Q_{1}}{w^{9} \sin ^{2} \lambda}
\end{aligned}
$$

As in the case of a D3 $\perp$ NS, we can map this solution to its S-dual by multiplying the metric by $H_{5}^{-1 / 2}$ and taking $\phi \rightarrow-\phi$. We can also T-dualize to get a D0 smeared in the 1 direction and localized on an orbifold singularity. In [19] it was speculated that the solution of N D0 is dual to super-symmetric quantum mechanics. 


\section{Semi-localized Dp branes}

We now turn to configurations in which the Dp brane is smeared in the world volume of the NS brane. We shall find an explicit solution for D1 $\perp$ NS. Solutions for Dp-branes up to $\mathrm{p}=4$ will follow by $\mathrm{T}$ duality.

In the following construction the NS brane spans (12345), and is completely localized in (6789). The D1 (6) is localized in (789), but is smeared in (12345). Thus the harmonic function of the D1 brane does not depend on $x_{12345}$ and should obey $\Delta_{789} H_{1}=0$. This gives $H_{1}=1+Q_{1} / r$. In the near horizon limit we neglect the 1 in $H_{1}$. Thus $H_{5}$ satisfies a curved space Laplace equation

$$
\left[\Delta_{6}+H_{1}^{-1} \Delta_{789}\right] H_{5}(x, r)=0
$$

By defining $u=2 \sqrt{Q_{1} r}$ we get

$$
\left[\partial_{6}^{2}+u^{-3} \partial_{u}\left(u^{3} \partial_{u}\right)\right] H_{5}(x, u)=0
$$

Eq.(28) can be written using $w^{2}=x_{6}^{2}+u^{2}$ and $\tan \lambda=u / x_{6}$.

$$
\left[w^{-4} \partial_{w}\left(w^{4} \partial_{w}\right)+w^{-2} \Lambda(\lambda)\right] H_{5}(w, \lambda)=0
$$

We shall concentrate on solutions that have no $\lambda$ dependence, $H_{5}=1+$ $Q_{5} / w^{3}$. This solution can be generalized to

$$
H_{5}=1+\sum_{i=1}^{N_{5}} \frac{\mu_{i}}{\left(\left(x-x_{i}\right)^{2}+4 Q_{1} r\right)^{3 / 2}}
$$

The full SUGRA solution is according to eq.(1) :

$$
\begin{aligned}
d s^{2} & =\frac{w \sin \lambda}{2 Q_{1}} d x_{0}^{2}+\frac{2 Q_{1}}{w \sin \lambda} d x_{12345}^{2}+\frac{Q_{5} \sin \lambda}{2 Q_{1} w^{2}} d x_{6}^{2}+\frac{2 Q_{1} Q_{5}}{w^{4} \sin \lambda} d x_{789}^{2} \\
e^{2\left(\phi-\phi_{\infty}\right)} & =H_{5} H_{1}=\frac{4 Q_{1}^{2} Q_{5}}{w^{5} \sin ^{2} \lambda}
\end{aligned}
$$

It can be seen that the procedure will be the same for all other Dp branes up to $\mathrm{p}=4$. This is equivalent to T-dualizing along (12345). The effect of this duality is just $g_{i i} \rightarrow 1 / g_{i i}$. The case of an NS localized within a D6 brane was considered from another point of view in [9, 13]. It is also related by T-duality to the solutions shown here. Since in these solution the NSbrane is localized, we expect the corresponding field theory to live on the NS-brane. This field theory will have a RR background from the Dp-branes, and 8 supersymmetries. We shall not explore such field theories here. 


\section{Summary and discussion}

We have displayed solutions of semi-localized intersecting NS and Dp-branes. Given the metrics of these intersections, one can learn about the field theory duals to these configurations by calculating Wilson loops. For example, When calculating the Wilson loop in the NS $\perp$ D4 case, the metric factors into $A d S_{5} \times X^{5}$. Thus, provided that we do not move on $X^{5}$, i.e. change $\lambda$, the result will be independent of the nature of $X^{5}$. The same is also true for the rest of the configurations, that the Wilson loop of the field theory is determined by the part in the metric corresponding to the brane world volume coordinates, while the number of supersymmetries and matter content are determined by the nature of $X$. The solutions for Dp-branes intersecting NS branes were shown to be dual to $\mathrm{D}(\mathrm{p}-1)$-branes on singular backgrounds.

Similar solutions for Dp-branes intersecting Dp-branes can be found using the techniques shown here. It is, however, not clear what field theories a general intersection describes.

Acknowledgments. I would like to thank my supervisors Prof. J. Sonnenschein and Prof. S. Yankielowicz for helpful discussions. I would also like to thank N. Itzhaki, D. Smith, and A. Fayyazuddin for their comments.

\section{References}

[1] Jerome P. Gauntlett, "Intersecting Branes", hep-th/9705011, and references therein

[2] J. P. Gauntlett, G. W. Gibbons, G. Papadopoulos, P. K. Townsend, "Hyper-Kahler manifolds and multiply-intersecting branes", Nucl.Phys. B500 (1997) 133-162, hep-th/9702202

[3] Joaquim Gomis, David Mateos, Joan Simn, Paul K. Townsend, "BraneIntersection Dynamics from Branes in Brane Backgrounds", Phys.Lett. B430 (1998) 231-236, hep-th/9803040

[4] A. A. Tseytlin, "Composite BPS configurations of p-branes in 10 and 11 dimensions", Class.Quant.Grav. 14 (1997) 2085-2105, hep-th/9702163 
[5] A. A. Tseytlin, "No-force condition and BPS combinations of p-branes in 11 and 10 dimensions", Nucl.Phys. B487 (1997) 141-154, hepth/9609212

[6] A. A. Tseytlin, "Harmonic superpositions of M-branes", Nucl.Phys. B475 (1996) 149-163, hep-th/9604035

[7] R. Argurio, F. Englert, L. Houart, "Intersection Rules for p-Branes", Phys.Lett. B398 (1997) 61-68, hep-th/9701042

[8] Harm Jan Boonstra, Bas Peeters, Kostas Skenderis, "Brane intersections, anti-de Sitter spacetimes and dual superconformal theories", Nucl.Phys. B533 (1998) 127-162, hep-th/9803231

[9] N. Itzhaki, A. A. Tseytlin, S. Yankielowicz, "Supergravity Solutions for Branes Localized Within Branes", Phys.Lett. B432 (1998) 298-304, hepth/9803103

[10] Akikazu Hashimoto, "Supergravity Solutions for Localized Intersections of Branes", hep-th/9812159

[11] Jose D. Edelstein, Liviu Tataru, Radu Tatar, "Rules for Localized Overlappings and Intersections of p-Branes", JHEP 9806 (1998) 003, hepth/9801049

[12] Klaus Behrndt, Eric Bergshoeff, Bert Janssen, "Intersecting D-Branes in ten and six dimensions", Phys.Rev. D55 (1997) 3785-3792, hepth/9604168

[13] Oskar Pelc, Ruud Siebelink, "The D2-D6 System and a Fibered AdS Geometry", hep-th/9902045

[14] Amihay Hanany, Edward Witten, "Type IIB Superstrings, BPS Monopoles, And Three-Dimensional Gauge Dynamics", Nucl.Phys. B492 (1997) 152-190, hep-th/9611230

[15] Edward Witten, "Solutions Of Four-Dimensional Field Theories Via M Theory", Nucl.Phys. B500 (1997) 3-42, hep-th/9703166

[16] A. Giveon, D. Kutasov, "Brane Dynamics and Gauge Theory", hepth/9802067 
[17] E. Bergshoeff, C. M. Hull, T. Ortin, "Duality in the Type-II Superstring Effective Action", Nucl.Phys. B451 (1995) 547-578, hep-th/9504081

[18] Juan M. Maldacena, "The Large N Limit of Superconformal Field Theories and Supergravity Authors: Juan M. Maldacena", Adv.Theor.Math.Phys. 2 (1998) 231-252, hep-th/9711200

[19] Nissan Itzhaki, Juan M. Maldacena, Jacob Sonnenschein, Shimon Yankielowicz, "Supergravity and The Large N Limit of Theories With Sixteen Supercharges", Phys.Rev. D58 (1998) 046004, hep-th/9802042

[20] S. Kachru, E. Silverstein, "4d Conformal Field Theories and Strings on Orbifolds", Phys.Rev.Lett. 80 (1998) 4855-4858, hep-th/9802183

[21] A. Lawrence, N. Nekrasov, C. Vafa, "On Conformal Theories in Four Dimensions", Nucl.Phys. B533 (1998) 199-209, hep-th/9803015

[22] Keshav Dasgupta, Sunil Mukhi, "Brane Constructions, Conifolds and M-Theory", hep-th/9811139

[23] Angel M. Uranga, "Brane Configurations for Branes at Conifolds", hepth/9811004

[24] Haisong Yang, "Localized Intersecting Brane Solutions of D=11 Supergravity", hep-th/9902128 